\title{
ESTUDOS SENSORIAIS PARA DETERMINAÇÃO DA VIDA DE PRATELEIRA DE BARRA DE CEREAIS COM PIPOCA DE SORGO
}

\author{
CAROLINE LIBOREIRO PAIVA ${ }^{1}$, VALÉRIA APARECIDA VIEIRA QUEIROZ² \\ e JOSÉ AVELINO SANTOS RODRIGUES ${ }^{2}$
}

${ }^{1} U F M G$, Belo Horizonte, MG, Brasil, carolinepaiva7@gmail.com

${ }^{2}$ Embrapa Milho e Sorgo, Sete Lagoas, MG, Brasil,valeria.vieira@embrapa.br, avelino.rodrigues@embrapa.br

Revista Brasileira de Milho e Sorgo, v.11, n.3, p. 302-311, 2012

\begin{abstract}
RESUMO - O objetivo do trabalho foi determinar a vida de prateleira de barras de cereais elaboradas com pipoca de sorgo, a partir de avaliações sensoriais realizadas por uma equipe treinada, tendo como parâmetro a qualidade global do produto. Os produtos armazenados em duas condições de temperatura $\left(30^{\circ} \mathrm{C}\right.$ e sob condição acelerada, $\left.40^{\circ} \mathrm{C}\right)$ foram avaliados em períodos pré-determinados durante 90 dias. Como alternativa, aplicou-se também um teste rápido de ordenação com consumidores comuns, com o objetivo de verificar se havia preferência pelo produto recém-elaborado em detrimento aos armazenados por 90 dias em condição acelerada. A vida de prateleira em condição acelerada foi estimada em 77 dias por meio dos dados obtidos da regressão linear dos valores da qualidade global, avaliada pelo painel sensorial e pelo tempo de estocagem. A vida de prateleira do produto a $30^{\circ} \mathrm{C}$ foi de 144 dias. Também foi possível concluir que não houve perda significativa da qualidade global do produto durante os 90 dias de armazenamento, segundo a percepção tanto de provadores treinados, quanto de consumidores comuns de barras de cereais.
\end{abstract}

Palavras-chave: Sorghum bicolor L., cereais em barra, estudo de estabilidade, análise sensorial.

\section{SENSORY STUDIES TO ESTIMATE SHELF-LIFE OF CEREAL BARS WITH SORGHUM POPCORN}

\begin{abstract}
The objective of this work was to determinate the shelf-life of cereal bars containing sorghum popcorn, considering sensory evaluations done by trained team having as a parameter the global quality of the product. The products stored under two conditions of temperature $\left(30^{\circ} \mathrm{C}\right.$ and under accelerated conditions, $\left.40^{\circ} \mathrm{C}\right)$ were evaluated in pre-defined intervals during 90 days. Alternatively, this study has also carried out a rapid ranking test with common consumers, with the objective to check out if there has been a preference for the freshly product compared to the ones stored by 90 days under accelerated conditions. The shelf life in accelerated conditions was estimated in 77 days through data obtained by linear regression of global quality values, evaluated by sensory panel, and storage time. The shelf-life of the product under $30^{\circ} \mathrm{C}$ was 144 days. In addition, no significant loss occurred in the global quality of the product during the 90 days of storage, according to the perception not only of the trained evaluators but also of the common consumers of cereal bars.
\end{abstract}

Key words: Sorghum bicolor L., cereal bars, stability study, sensory analysis. 
Já é consenso que a qualidade de vida está associada, entre outros fatores, à qualidade da dieta que se consome. Por isso, é crescente o interesse dos meios acadêmico e empresarial por um ramo da ciência e tecnologia de alimentos: o dos produtos funcionais. Estes alimentos são assim denominados porque, além das suas funções básicas relativas à nutrição, exercem atividade biológica e têm sido sugeridos como importantes meios de redução do risco de diversas doenças crônico-degenerativas, enfermidades inflamatórias e distúrbios metabólicos.

Neste contexto, pesquisadores vêm demonstrando que o sorgo integral, utilizado como base alimentar de milhões de pessoas, principalmente na África e na Ásia, além de fonte de carboidratos, é uma rica fonte de fibras e de compostos bioativos, incluindo taninos, ácidos fenólicos, antocianinas e fitoesteróis. Praticamente todas as espécies de sorgo contêm ácidos fenólicos, localizados no pericarpo, na testa, na camada de aleurona e no endosperma (Mcdonough et al., 1986).

Especulações a respeito da relação entre o consumo de sorgo e a prevenção de doenças cardiovasculares, obesidade e alguns tipos de câncer também começaram a surgir (Awika \& Rooney, 2004, Dicko et al., 2006). Estudos in vitro demonstraram que o sorgo de pericarpo preto induziu enzimas detoxificantes e aqueles com teores mais elevados de taninos inibiram a proliferação de células de câncer de esôfago e cólon. Os extratos de sorgo apresentaram forte potencial quimiopreventivo, independente da sua atividade antioxidante (Awika et al., 2009). Além disso, quantidades consideráveis de amido resistente, de fibra dietética, de fitoesteróis, de minerais, de tocoferóis e de tocotrienóis têm sido identificadas em diversas cultivares de sorgo (Nilsson et al., 1997; Glitsø \& Bach Knudsen, 1999; Shin, 2004).
Neste contexto, a Embrapa Milho e Sorgo iniciou em 2007 uma linha de pesquisa visando à incorporação do sorgo na alimentação da população brasileira, através do desenvolvimento de produtos. A barra de cereais com pipoca de sorgo foi um dos produtos desenvolvidos. A análise sensorial do produto, por meio de teste afetivo de aceitação, comprovou $100 \%$ de aceitação em relação aos atributos de aparência e sabor e 92,5 e 96,3 \% quanto à cor e à textura, respectivamente (Queiroz et al., 2008). No entanto, a vida de prateleira do produto ainda não havia sido testada.

O desenvolvimento de barras de cereais se justifica pelo constante crescimento no consumo deste produto, devido principalmente à sua conveniência e à associação como alimento saudável (Sampaio et al., 2009). Além disto, vários estudos destacam o problema da baixa ingestão de fibras pela população brasileira, sendo, então, a barra de cereais um produto que promoveria o aumento da ingestão de fibras, reduzindo o problema de constipação intestinal em adultos e crianças (Madruga et al., 2009; Neutzling et al., 2010).

O estudo da vida de prateleira de um determinado produto consiste em submeter várias amostras deste produto, em períodos pré-definidos, a testes físico-químicos, sensoriais ou microbiológicos capazes de identificar a perda de qualidade do alimento (Netto, 2010). A vida de prateleira é definida, então, pelo período de armazenamento em que o produto com qualidade adequada permanece próprio para consumo sob condições estabelecidas de temperatura, umidade relativa, luz e outras, sofrendo pequenas alterações que não afetam a sua qualidade sensorial, nutricional e a segurança do consumidor (Vitali et al., 2010a). Para estimar a vida de prateleira, é comum aplicar testes acelerados. Nestes testes, 
as amostras do produto são submetidas a condições forçadas de armazenamento a fim de acelerar a degradação química e/ou mudanças físicas. Através de modelos matemáticos, é possível predizer, então, a vida de prateleira do produto em temperaturas inferiores.

Diferentes testes sensoriais também podem ser aplicados em estudos de determinação da vida de prateleira de produtos alimentícios. Testes sensoriais descritivos são úteis para avaliar diferenças de intensidade de atributos sensoriais de interesse ao longo do período de armazenamento do produto (Garcia, 2010). Tais testes têm a vantagem de serem confiáveis e consistentes por empregarem julgadores selecionados e treinados, mas podem ser demorados e caros por demandarem treinamento longo (Richter, 2006). De outra forma, Hough et al. (2003) já indicavam que estudos sensoriais com consumidores são mais interessantes pelo fato de serem estes os que, na realidade, decidem pela aceitação do produto depois de certo tempo de estocagem.

Assim, o presente trabalho teve como objetivo determinar a vida de prateleira de barras de cereais elaboradas com pipoca de sorgo a partir de avaliações sensoriais realizadas por uma equipe treinada, tendo como parâmetro a qualidade global do produto.

\section{Material e Métodos}

\section{Elaboração das barras de cereais}

O experimento foi realizado no Laboratório de Desenvolvimento de Novos Produtos do Centro Universitário de Belo Horizonte (UNI-BH) no segundo semestre de 2010. Para a produção das barras de cereais com pipoca de sorgo, foram utilizados aveia, flocos de arroz, canela em pó, banana desidratada, glicose de milho, açúcar mascavo, gordura de palma, lecitina de soja, açúcar invertido e pipoca de sorgo (BRS 309 - Embrapa Milho e Sorgo, Sete Lagoas, MG), conforme metodologia descrita por Queiroz et al. (2008).

Após a elaboração, as barras de cereais foram desenformadas, cortadas em tamanhos retangulares e acondicionadas individualmente em embalagens de filme flexível PET/PEBD/AL/PEBD (politereftalato de etileno, polietileno de baixa densidade, película de folha de alumínio e polietileno de baixa densidade), fornecido por Shellmar Embalagens Modernas Ltda. As barras de cereais elaboradas com pipoca de sorgo foram, então, submetidas à análise de fibra alimentar, às análises microbiológicas de coliformes a $45{ }^{\circ} \mathrm{C}$, Bacillus cereus e Salmonella sp., e aos testes sensoriais para a estimativa da vida de prateleira.

\section{Estimativa da vida de prateleira}

O delineamento experimental empregado foi o inteiramente casualizado, com dois tratamentos: barra de cereais armazenada em duas condições de temperatura, $30^{\circ} \mathrm{C}$, temperatura ambiente, e sob condição acelerada, $40^{\circ} \mathrm{C}$, conforme proposto por Vitali et al. (2010) para alimentos com teor intermediário de umidade. O período de estudo foi de 90 dias, sendo a primeira amostragem realizada no $15^{\circ}$ dia e as seguintes a cada 30 dias a partir da elaboração do produto.

A estimativa da vida de prateleira foi estabelecida com base nas respostas da avaliação sensorial por uma equipe treinada em relação à qualidade global do produto em função do tempo de armazenamento, por regressão linear simples, mediante programa Excel 2003. A nota 4,5 (não gostei/nem desgostei) da escala de qualidade global do teste sensorial foi o ponto de corte que definiu o fim da vida útil da barra de cereais (Garcia, 2010). 


\section{Análises sensoriais}

Para a avaliação da vida de prateleira da barra de cereais com sorgo, foram feitos dois experimentos: um com uma equipe treinada de sete provadores e outro com 42 consumidores comuns, não treinados.

Inicialmente, empregou-se um teste de ordenação para provadores não treinados. Quatro produtos foram apresentados a 42 pessoas: a barra de cereais com pipoca de sorgo recém-elaborada e também as armazenadas por 30, 60 e 90 dias, todas a $40{ }^{\circ} \mathrm{C}$. Aos provadores, foi solicitado que ordenassem as amostras da mais preferida à menos preferida, tendo-se atribuído o valor 1 à mais preferida e o valor 4 à menos preferida. Para a execução dos testes, as amostras de aproximadamente $15 \mathrm{~g}$ foram apresentadas aos julgadores, todas de uma só vez, em guardanapos sobre bandejas codificadas com números de três dígitos aleatórios. Durante os testes sensoriais, foi disponibilizada água aos provadores e solicitado que enxaguassem a boca após degustarem cada amostra. A avaliação dos resultados foi realizada por meio do Teste de Friedmam a 5 \% de significância. O teste de Friedman é utilizado para avaliar respostas de uma variável categórica ordinal (ordem da preferência) em subgrupos dependentes (cada provador experimentou amostras armazenadas no tempo 0, 30, 60 e 90 dias) (Siegel \& Castellan, 2006).

Com o objetivo de verificar a qualidade global do produto e as alterações de brilho, textura e sabor, utilizou-se uma equipe de sete provadores previamente selecionados após responderem a uma ficha convite e participarem de testes para avaliação da acuidade sensorial, além de vários treinamentos. Após pré-seleção, os provadores participaram de testes discriminativos, especificamente de dez testes triangulares em dias alternados com diferentes produtos alimentícios, de acordo com metodologia proposta por Faria \& Yotsuyanagi (2008).

Para avaliar a capacidade de detectarem diferenças de intensidade, foram aplicados testes de gostos primários, limiar absoluto e ordenação, usando soluções de sacarose, ácido cítrico, cafeína e cloreto de sódio em diferentes concentrações. Os candidatos que atenderam aos critérios de aprovação para verificação da acuidade sensorial se reuniram, então, para definir os descritores dos atributos sensoriais da barra de cereais com pipoca de sorgo. Uma lista de definição de cada termo descritivo foi elaborada e materiais de referência foram sugeridos pela equipe. O objetivo foi desenvolver a ficha de avaliação sensorial com as escalas para expressar a intensidade percebida dos descritores já consensados pela equipe de provadores. Isso foi realizado em sete sessões por meio de discussão e degustação do produto. Em seguida, durante oito sessões, fez-se o treinamento nos atributos e no uso das escalas através de degustação das barras de cereais em cabines individuais (Faria \& Yotsuyanagi, 2008). Os avaliadores selecionados foram os que apresentaram melhor memória sensorial, habilidade e reprodutibilidade das respostas, medidas através da tabulação e do desvio padrão dos dados obtidos durante o treinamento.

Os avaliadores foram conduzidos, então, para os testes sensoriais que definiriam a vida de prateleira do produto. As amostras foram apresentadas em pratos plásticos, marcados com uma combinação de três números aleatórios, codificados de forma a corresponder a uma determinada temperatura de armazenamento $\left(30\right.$ e $40{ }^{\circ} \mathrm{C}$ ). Nos pratos, foi colocada uma amostra de $20 \mathrm{~g}$ de forma inteiramente casualizada. Empregou-se escala não estruturada de $9 \mathrm{~cm}$ para indicar a intensidade dos atributos de brilho (ruim a excelente), textura (ruim a característica), sabor 
(característico a estranho) e qualidade global (ruim a excelente). As análises foram feitas no produto recém-elaborado e nos armazenados por 15, 30, 60 e 90 dias. Os resultados foram avaliados estatisticamente por análise de variância (Anova) e as médias dos atributos foram comparadas pelo teste de Tukey.

\section{Determinação de fibra}

As barras de cereais, logo após a sua elaboração, foram caracterizadas quanto à composição de fibra alimentar segundo procedimento da Association (2005), método número 985.29. O objetivo foi verificar se o produto poderia ser considerado fonte de fibras.

\section{Avaliações microbiológicas}

As determinações de Bacillus cereus e coliformes a $45{ }^{\circ} \mathrm{C}$ e a pesquisa de Salmonella sp. foram realizadas de acordo com a metodologia proposta pelos métodos oficiais da Association (2005), métodos números 17.8.01, 17.2.02 e 989.13, respectivamente.

As análises microbiológicas foram realizadas nos produtos logo após a elaboração e, nos armazenados por 90 dias, a $30^{\circ} \mathrm{C}$ e a $40{ }^{\circ} \mathrm{C}$ em três repetições.

\section{Resultados e Discussão}

\section{Avaliações sensoriais}

A Tabela 1 descreve os resultados dos testes sensoriais realizados pela equipe de sete provadores treinados que degustaram as barras de cereais com pipoca de sorgo armazenadas a 30 e a $40{ }^{\circ} \mathrm{C}$ durante 90 dias. Os provadores não perceberam diferenças significativas em nenhum atributo de qualidade avaliado nas barras de cereais armazenadas a $30^{\circ} \mathrm{C}$ durante o período de armazenamento (Tabela 1). No entanto, nas barras de cereais armazenadas a $40^{\circ} \mathrm{C}$, a partir de 90 dias de estocagem, foram percebidas perdas significativas da qualidade global e de textura e perda

TABELA 1. Média dos escores de sabor, qualidade global, brilho e textura da barra de cereais com pipoca de sorgo ${ }^{1}$.

\begin{tabular}{|c|c|c|c|c|c|c|}
\hline \multirow[t]{2}{*}{ Atributo } & \multirow{2}{*}{$\begin{array}{l}\text { Temperatura de } \\
\text { armazenamento } \\
\left({ }^{\circ} \mathrm{C}\right)\end{array}$} & \multicolumn{5}{|c|}{ Tempo em dias } \\
\hline & & 0 & 15 & 30 & 60 & 90 \\
\hline \multirow{2}{*}{ Sabor $^{2}$} & 30 & $8,72 \mathrm{Aa}$ & 7,48 Aa & $8,00 \mathrm{Aa}$ & $6,86 \mathrm{Aa}$ & $7,28 \mathrm{Aa}$ \\
\hline & 40 & $8,72 \mathrm{Aa}$ & $7,38 \mathrm{Aa}$ & $7,20 \mathrm{Aa}$ & $6,82 \mathrm{Aa}$ & $6,30 \mathrm{Aa}$ \\
\hline \multirow{2}{*}{ Qualidade global ${ }^{3}$} & 30 & $7,58 \mathrm{Aa}$ & $6,54 \mathrm{Aa}$ & $7,02 \mathrm{Aa}$ & $5,58 \mathrm{Aa}$ & $5,88 \mathrm{Aa}$ \\
\hline & 40 & $7,58 \mathrm{Aa}$ & $6,60 \mathrm{Aa}$ & $6,20 \mathrm{Aa}$ & $4,97 \mathrm{Aa}$ & $4,20 \mathrm{Bb}$ \\
\hline \multirow{2}{*}{ Brilho $^{4}$} & 30 & $5,86 \mathrm{Aa}$ & 7,02 Aa & $5,12 \mathrm{Aa}$ & $5,02 \mathrm{Aa}$ & $4,54 \mathrm{Aa}$ \\
\hline & 40 & $5,86 \mathrm{Aa}$ & $6,92 \mathrm{Aa}$ & $4,14 \mathrm{Ab}$ & $4,04 \mathrm{Bb}$ & $3,74 \mathrm{Ab}$ \\
\hline \multirow{2}{*}{ Textura ${ }^{5}$} & 30 & $6,52 \mathrm{Aa}$ & $6,72 \mathrm{Aa}$ & $6,48 \mathrm{Aa}$ & $4,64 \mathrm{Aa}$ & $4,92 \mathrm{Aa}$ \\
\hline & 40 & $6,52 \mathrm{Aa}$ & $6,14 \mathrm{Aa}$ & $5,64 \mathrm{Aa}$ & $4,66 \mathrm{Aa}$ & $3,24 \mathrm{Bb}$ \\
\hline
\end{tabular}


significativa de brilho a partir de 30 dias. Em relação ao sabor, não houve nenhuma perda significativa para as barras armazenadas a $40^{\circ} \mathrm{C}$.

A Tabela 2 apresenta o resultado da análise sensorial discriminativa aplicada ao grupo de provadores não treinados. Valor 1 e valor 4 foram atribuídos à amostra mais e menos preferida, respectivamente. Analisando-se os resultados, verifica-se que os consumidores não perceberam nenhuma diferença significativa entre as barras de cereais armazenadas ao longo dos 90 dias à temperatura extrema de $40^{\circ} \mathrm{C}$. percepção do consumidor, como afirma Hough et al. (2003). Os testes de ordenação têm sido utilizados em estudos de preferência com consumidores por ser uma técnica simples (Faria \& Yotsuyanagi, 2008). Lee \& O'Mahony (2005) propuseram testes de ordenação como alternativa mais simples que as escalas de intensidade, sob o argumento de que os consumidores não estão acostumados ou podem não concordar com as categorias da escala empregada. No entanto, este resultado não foi integralmente comprovado pelo teste descritivo aplicado ao grupo de provadores treina-

TABELA 2. Mediana das ordens de preferência em relação às barras de cereais com pipoca de sorgo armazenadas a $40{ }^{\circ} \mathrm{C}^{1}$.

\begin{tabular}{cc}
\hline Tempo (dias) & Mediana da Ordem de preferência \\
\hline 0 & $2,0 \mathrm{~A}$ \\
30 & $3,0 \mathrm{~A}$ \\
60 & $2,5 \mathrm{~A}$ \\
90 & $3,0 \mathrm{~A}$ \\
\hline
\end{tabular}

${ }^{1}$ Medianas com letras iguais não diferem significativamente entre si pelo teste de Friedman a 5\%.

Embora os testes descritivos sejam mais consistentes, há uma grande desvantagem pelo fato das análises exigirem acuidade sensorial da equipe, treinamento nos atributos a serem avaliados e concordância dos resultados, o que torna o método demorado e caro. Normalmente, há a necessidade de se recrutar um grande número de provadores, pois muitos são desclassificados ou desistem diante do grande número de sessões (Richter, 2006; Faria \& Yotsuyanagi, 2008). Este fato foi observado no presente estudo, no qual, dos 48 voluntários recrutados para os testes de acuidade, somente sete realizaram os testes sensoriais de determinação da vida de prateleira.

A proposta do teste com consumidores realizada neste estudo leva em consideração que a vida de prateleira de produtos alimentícios depende da dos, pois estes não perceberam perdas significativas de textura, brilho e qualidade global para as barras de cereais armazenadas a $40^{\circ} \mathrm{C}$ ao longo do período de armazenamento. Segundo o teste descritivo, só para as barras armazenadas a $30^{\circ} \mathrm{C}$ não houve percepção de perda de qualidade, como já relatado. Este resultado sugere que, para estudos de vida de prateleira, testes mais robustos devem ser aplicados a consumidores comuns.

Em relação ao estudo da vida de prateleira da barra de cereais com pipoca de sorgo, os dados obtidos por meio de regressão linear com os valores da qualidade global, avaliada pelo painel sensorial e pelo tempo de estocagem nas duas condições de temperatura, mostram que a reação de perda da qualidade sensorial se ajusta ao modelo cinético de ordem zero (Figura 1). 


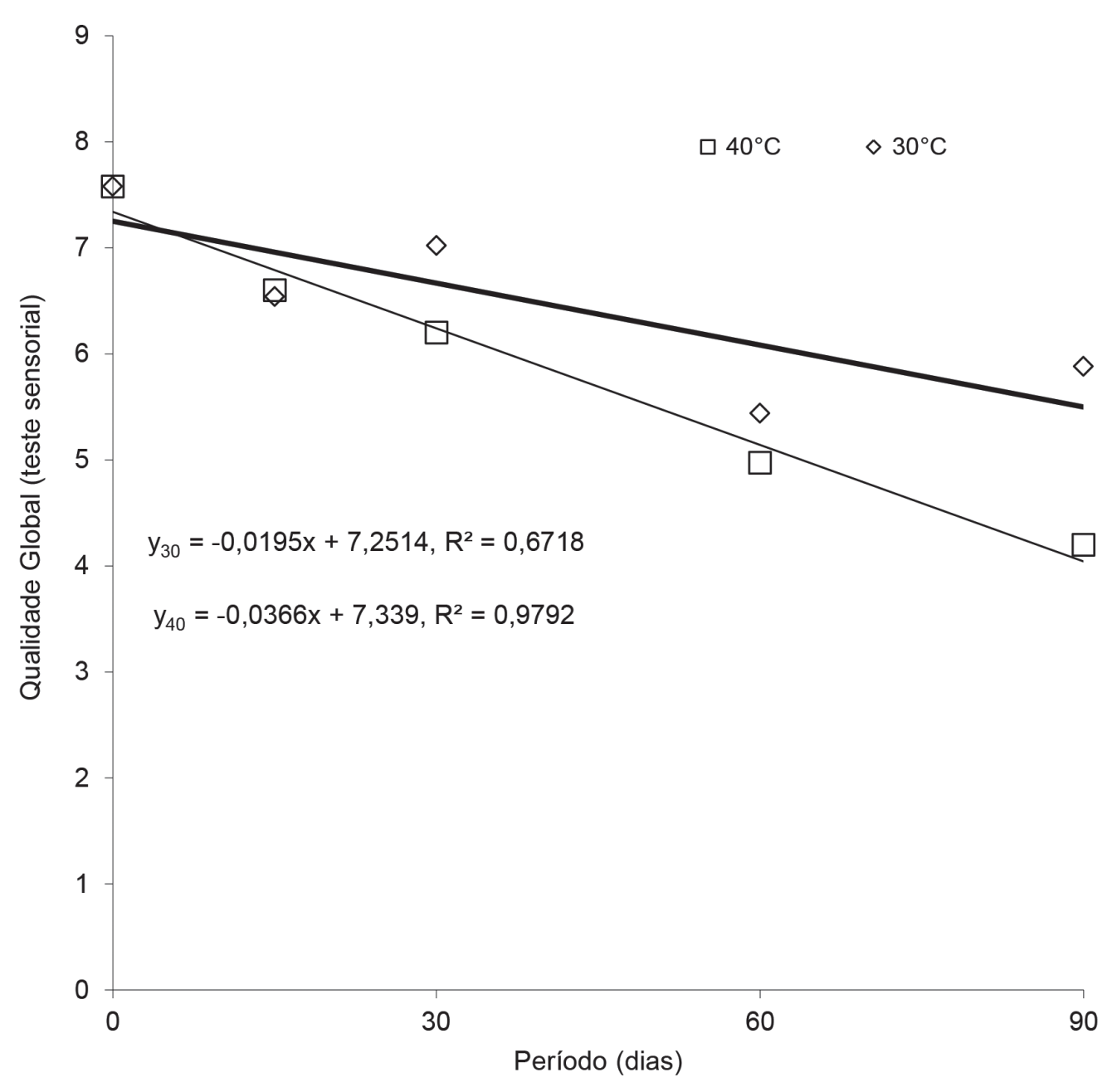

FIGURA 1. Modelo cinético da qualidade global da barra de cereais com pipoca de sorgo armazenada a $30{ }^{\circ} \mathrm{C}$ e a $40{ }^{\circ} \mathrm{C}$ durante 90 dias.

A vida de prateleira da barra de cereais armazenada sob condição acelerada $\left(40^{\circ} \mathrm{C}\right)$ foi determinada, então, em 77 dias, período em que a qualidade global atingiu o valor de 4,5. Este ponto de corte também foi utilizado por Grizotto et al. (2006) e sugerido por Garcia (2010). O coeficiente de determinação $\left(\mathrm{R}^{2}=0,98\right)$ indica que o modelo é adequado. Segundo Villanueva \& Trindade (2009), coeficientes de determinação acima de 0,6 são considerados bons para estudos sensoriais.
Relações lineares entre a qualidade global e o tempo de estocagem também foram encontrados por Grizotto et al. (2006) no estudo da vida de prateleira de fruta estruturada de polpa concentrada de mamão. Da mesma forma, Gámbaro et al. (2004) também encontraram relação linear entre atributo sensorial, avaliado por equipe treinada, versus tempo de estocagem de alfajor, espécie de bolo bastante consumido em países como Argentina e Uruguai. 
A partir do modelo cinético (Figura 1), calculou-se inicialmente o valor de $\mathrm{Q}_{10}$ através do quociente entre as velocidades de reação $(\mathrm{k})$ a 30 e a $40{ }^{\circ} \mathrm{C}$. Em seguida, como indicado por Vitali et al. (2010b), a vida de prateleira a $30{ }^{\circ} \mathrm{C}$ foi determinada em 144 através da Equação 1:

$\mathrm{Q}_{10}=\mathrm{VP}_{1} / \mathrm{VP}_{2}$

Onde:

$\mathrm{VP}_{1}=$ vida de prateleira a $30^{\circ} \mathrm{C}$ e $\mathrm{VP}_{2}=$ vida de prateleira a $40{ }^{\circ} \mathrm{C}$ (77 dias).

$\mathrm{O}$ valor de $\mathrm{Q}_{10}$ igual a 1,9 é compatível com os valores para qualidade sensorial global de produtos de origem vegetal, citado por Vitali et al. (2010b).

\section{Avaliações microbiológicas}

As análises de Bacilus cereus, coliformes a 45 ${ }^{\circ} \mathrm{C}$ e Salmonella mostraram que não houve crescimento destes microrganismos nas barras elaboradas e armazenadas a 30 e a $40{ }^{\circ} \mathrm{C}$, ao longo de 90 dias, indicando adequada qualidade microbiológica para consumo.

\section{Avaliação de fibra}

O resultado de fibra alimentar encontrado foi de 5,40g/100g do produto. Segundo a Portaria 27 da Agência Nacional de Vigilância Sanitária, de 13 de janeiro de 1998, o valor caracteriza o produto como fonte de fibras, por possuir mais de $1,5 \mathrm{~g}$ de fibra por $100 \mathrm{~g}$ do produto (Brasil, 1998).

\section{Conclusões}

O desenvolvimento deste trabalho possibilitou estabelecer a vida de prateleira de 144 dias para o produto barra de cereais com pipoca de sorgo, compatível com o prazo de validade de diversas barras elaboradas sem aditivos. Além disso, verificou-se que o emprego de análises sensoriais com equipe treinada é ferramenta importante na determinação da vida de prateleira do produto desenvolvido.

Verificou-se ainda que o produto pode ser uma alternativa de consumo para pessoas preocupadas em atender à recomendação de ingestão de alimentos com teor aumentado de fibras dietéticas.

\section{Agradecimentos}

Ao UNI-BH, por ter cedido as suas instalações para a elaboração e a embalagem do produto.

\section{Referências}

ASSOCIATION OF OFFICIAL ANALYTICAL CHEMISTS. Official methods of analysis of AOAC International. 18. ed. atual. Gaithersburg, MA: AOAC International, 2005.

AWIKA, J. M.; ROONEY, L.W. Sorghum phytochemicals and their potential impact on human health. Phytochemistry. New York, v. 65, p. 1199-1221, 2004.

AWIKA, J. M.; YANG, L.; BROWNING, J. D.; FARAJ, A. Comparative antioxidant, antiproliferative and phase II enzyme inducing potential of sorghum (Sorghum bicolor) varieties. LWT - Food Science and Technology, Heights, v. 42, p. 1041-1046, 2009.

BRASIL. Portaria nº 27, de 13 de janeiro de 1998. Agência Nacional de Vigilância Sanitária. Regulamento Técnico referente a informação nutricional complementar. Diário Oficial da União, Brasília, DF. Disponível em <http://www.anvisa.gov.br>. Acesso em: 30 jun. 2011.

DICKO, M. H.; GRUPPEN, H.; TRAORÉ, A. S.; VORAGEN, A. G. J.; VAN BERKEL, W. J. H. Sorghum grain 
as human food in Africa: relevance of content of starch and amylase activities- review. African Journal of Biotechnology, Nairobi, v. 5, n. 5, p. 384-395, 2006.

FARIA, E. V.; YOTSUYANAGI, K. Técnicas de análise sensorial. 2. ed. Campinas: ITAL, 2008. 120 p.

GÁMBARO, A.; GIMÉNEZ, A.; VARELA, P. Sensory shelf-life estimation of alfajour by survival analysis. Journal of Sensory Studies, Westport, v. 19, p. 500509, 2004.

GARCIA, A. O. Determinação da vida-de-prateleira pela análise sensorial. In: MOURA, S. C. S. R.; GERMER, S. P. M. Reações de transformação e vida-de-prateleira de alimentos processados. 4. ed. Campinas: ITAL, 2010. p. 64-79.

GLITS $\varnothing$, L.V.; BACH KNUDSEN, K.E. Milling of whole grain rye to obtain fractions with different dietary fibre characteristics. Journal of Cereal Science, London, v. 29, p. 89-97, 1999.

GRIZOTTO, R.K.; BERBARI, S.A.G.; MOURA, S.C.S.R.; CLAUS, M.L. Estudo da vida-de-prateleira de fruta estruturada desidratada obtida de polpa congelada de mamão. Ciência e Tecnologia de Alimentos, Campinas, v. 26, n. 3, p. 709-714, 2006.

HOUGH,G.; LANGOUHR, K.; GÓMEZ, G.; CURIA, A. Survival analysis applied to sensory shelf life of foods. Journal of Food Science, London, v. 68, n. 1, p. 359 362, 2003.

LEE, H. S; O’MAHONY, M. Sensory evaluation and marketing: measurement of a consumer concept. Food Quality and Preference, New York, v.16, p. 227-235, 2005. MADRUGA, S. W.; ARAÚJJ, C. L.; BERTOLDI, A. D. Frequency of fi ber-rich food intake and associated factors in a Southern Brazilian population. Caderno de Saúde Pública, Rio de Janeiro, v. 25, n.10, p. 2249$2259,2009$.

MCDONOUGH, C. M.; ROONEY, L. W.; EARP, C. F. Structural characteristics of Eleusine coracana (finger millet) using scanning electron and fluorescence microscopy. Food Microstructure, O’hare, v. 5, p. 247256, 1986.

NETTO, F. M. Determinação da vida-de-prateleira - Erros e limitações. In: MOURA, S. C. S. R.; GERMER, S. P. M. Reações de transformação e vida-de-prateleira de alimentos processados. 4. ed. Campinas: ITAL, 2010. p. 88-96

NEUTZLING, M. B. N.; ARAÚJO, C. L.; VIEIRA, M. F. A.; HALLAL, P. C.; MENEZES, A. M. B.; VICTORA, C. G. Intake of fat and fi ber-rich foods according to socioeconomic status: the 11-year follow-up of the 1993 Pelotas (Brazil) birth cohort study. Caderno de Saúde Pública, Rio de Janeiro, v. 26, n.10, p. 1904$1911,2010$.

NILSSON, M.; AMAN, P.; HÄRKÖNEN, H.; HALLMANS, G.; KNUDSEN, K .E. B.; MAZUR, W.; ADLERCREUTZ, H. Content of nutrients and lignans in roller milled fractions of rye. Journal of the Science of Food and Agriculture, London, v. 73, p. 143=148, 1997.

QUEIROZ, V. A. V.; CARNEIRO, H. L.; VASCONCELOS, J. H.; RODRIGUES, J. A. S. Análise sensorial de um protótipo de barra de cereais elaborada com pipoca de sorgo. Sete Lagoas: Embrapa Milho e Sorgo, 2008. 8 p. (Embrapa Milho e Sorgo. Comunicado Técnico, 164).

RICHTER, V. B. Desenvolvimento de uma técnica sensorial por ordenação. 2006. 74 f. Dissertação (Mestrado). Departamento de Tecnologia de Alimentos e Medicamentos, Universidade Estadual de Londrina, Londrina.

SAMPAIO, C .R. P.; FERREIRA, S. M. R.; CANNIATTI-BRAZACA, S. G. Perfil sensorial e aceitabilidade de barras de cereais fortificadas com ferro. Alimentos e Nutrição, Marilia, v.20, n.1, p. 95-106, 2009. 
SHIN, S. I.; CHOI, H. J.; CHUNG, K. M.; HAMAKER, B. R.; PARK, K. H.; MOON, T. W.. Slowly digestible starch from debranched waxy sorghum starch: Preparation and properties. Cereal Chemistry, St. Paul, v. 81, p. 404-408, 2004.

SIEGEL S.; CASTELLAN JR, N. J. Estatística não-paramétrica para ciências do comportamento. 2 ed. Porto Alegre: Artmed, 2006, 448 p.

VILLANUEVA, N. D. M.; TRINDADE, M. A. Estimating sensory shelf life of chocolate and carrot cupcakes using acceptance tests. Journal of Sensory Studies, Westport, v. 25, p. 260-279, 2009.
VITALI, A. A.; TEIXEIRA NETO, R. O.; MOURA, S. C. S. R. Introdução à cinética de reação em alimentos. In: MOURA, S. C. S. R.; GERMER, S. P. M. Reações de transformação e vida-de-prateleira de alimentos processados. 4. ed. Campinas: ITAL, 2010a. p. 24-46.

VITALI, A. A.; TEIXEIRA NETO, R. O.; GERMER, S. P. M. Testes Acelerados de vida-de-prateleira de alimentos. In: MOURA, S. C. S. R.; GERMER, S. P. M. Reações de transformação e vida-de-prateleira de alimentos processados. 4. ed. Campinas: ITAL, 2010b. p. 80-87. 